\title{
Double cosets in free groups
}

\author{
Elizaveta Frenkel \\ Vladimir N. Remeslennikov
}

September 18, 2018

\begin{abstract}
In this paper we study double cosets of finite rank free groups. We focus our attention on cancellation types in double cosets and their formal language properties.
\end{abstract}

Mathematics Subject Classification 2010: 20E05, 20F10, 20F65

Key words: double cosets, subgroups of free groups, regular subsets, bounded cancellations.

\section{Introduction}

By the classical Nielsen-Schreier theorem every subgroup of a free group is again a free group. One of the goals of this paper is to study how one finitely generated free group $C$ can be embedded into another free group $F$. Clearly, the type of embedding of a subgroup $C$ into a group $F$ affects left and right cosets, and double cosets of type $C f C, f \in F$. A description of combinatorial and formal language properties of these complexes is the second goal of the present paper.

Similar questions have been studied widely as auxiliary ones, for example, in papers [3], [13, [16] in relation to hyperbolicity of amalgamated products of groups, and in [4, 8], 9] in connection with the conjugacy problem in amalgamated products.

There exist a lot of papers about the characterization of embeddings of a subgroup $C$ in a group $F$, but we shall mention only the closest to the subject of our paper. In [1] was shown that the property of a finitely generated subgroup $C$ to be malnormal is algorithmically decidable in a free group $F$, meanwhile [5] provides an example of the undecidability of this problem in the class of hyperbolic groups. Another related result was 
proved in [11, where it was shown that a random finitely generated subgroup of a free group $F$ is malnormal.

The essential role in these studies is played by the structure of a Cayley graph of a group and Schreier graphs for a subgroup $C$ in $F$. In particular, the embedding of $C$ in $F$ can be described in terms of equations $E(f, f)$ and $E(f, g)$ (see section 3.1), lemmas 3.1, 3.4). In this paper we obtain results on double cosets in a free group $F$ with respect to the type of embedding of a subgroup $C$ in $F$. The main results are the following theorems:

Theorem 3.12, Let $F$ be a free group with a finite basis $X$, and $C$ be a finitely generated subgroup of $F$ with a Nielsen set of generators $Y$. Suppose $f \in F \backslash C$ and $f$ is of minimal length in a double coset $C f C$. Then there exists a natural number $k$ which can be effectively determined by $C$ and $Y$ such that

- if $C_{f}=C \bigcap f^{-1} C f=1$, then multiplication in the complex $C f C$ is $k$-reduced, which means $l_{X}\left(c_{1}\right)+l_{X}(f)+l_{X}\left(c_{2}\right)-l_{X}\left(c_{1} f c_{2}\right) \leq 2 k$, where $c_{1}, c_{2} \in C$ and $l_{X}(\cdot)$ is the length of elements in $F(X)$.

- if $C_{f} \neq 1$, then $C f T$ is $k$-reduced, where $T$ is a relative Schreier transversal of $C$ in $F$ relative to $C_{f}$.

Theorem 3.16. Let $C$ be a finite rank subgroup of a free group $F(X)$. Then the set of all $X$-reduced words representing elements of $C f C$ is regular in $F(X)$. Moreover, an automaton which accepts this set can be constructed effectively by $C$ and $f$ if $C$ is $f$-malnormal, or by $C, f$ and a relative Schreier transversal $T$ of $C$ with respect to $C_{f}$, otherwise.

\section{Preliminaries}

In this section, following [7, 8, 12, we give some basics on regular subsets, cosets, and Schreier representatives of finitely generated subgroups of a finite rank free group $F$. We shall use the following notation throughout the paper.

Let $F=F(X)$ be a free group with basis $X=\left\{x_{1}, \ldots, x_{m}\right\}$. We identify elements of $F$ with reduced words in the alphabet $X \cup X^{-1}$ (i.e. $X$-reduced words), and let $l_{X}(f)$ denote the length of an element $f \in F$, i.e. the number of letters of the reduced word $f$ in $X \cup X^{-1}$.

Fix a subgroup $C=\left\langle h_{1}, \ldots, h_{r}\right\rangle$ of $F$ generated by finitely many elements $h_{1}, \ldots, h_{r} \in F$ and an arbitrary element $f \in F$.

The set $C f C=\left\{c_{1} f c_{2} \mid c_{1}, c_{2} \in C\right\}$ is called a double coset and $f$ is a representative of this coset. 


\subsection{Regular subsets in a free group and finite state automata}

Suppose we are given a finite set $X$, which we shall call the state set. An arrow is a triple $\left(s_{1}, x, s_{2}\right)$, where $s_{1}, s_{2}$ are elements of $S(\mathcal{A})$ and $x$ is an element of $X \cup \varepsilon$ and is called the label of the arrow. The source of the arrow is $s_{1}$ and its target is $s_{2}$. An arrow labelled $x$ is sometimes called an $x$-transition.

A non-deterministic finite state automaton $\mathcal{A}$ is a quintuple $\left(S(\mathcal{A}), X, \delta, S_{0}, F_{0}\right)$, where $S(\mathcal{A})$ is a finite set of states, $X$ is a finite set called the alphabet, $S_{0} \subset S(\mathcal{A})$ is the (non-empty) set of initial states, $F_{0} \subseteq S(\mathcal{A})$ is the set of final states, and $\delta$ is a set of arrows with labels in the enlarged alphabet $X \cup \varepsilon$. Here $\varepsilon$ is assumed not to lie in $X$.

By a path of arrows in $\mathcal{A}$ we mean a sequence $\left(s_{1}, u_{1}, s_{2}, \ldots, u_{n}, s_{n+1}\right)$, where $n \geq 0$ and each $u_{i}$ is an arrow with source $s_{i}$ and target $s_{i+1}$, for $1 \leq i \leq n$. We call $s_{1}$ the source and $s_{n+1}$ the target of the path of arrows. Let $w_{i}$ be the label of $u_{i}$, i.e. a letter of $X$ or $\varepsilon$. Let $w$ be the concatenation $w_{1} \cdots w_{n}$; if $n=0$, set $w=\varepsilon$. Then $w$ is called the label of the path. Let $X^{*}$ be a monoid of strings over $X$ with identity $\varepsilon$. The language $L(\mathcal{A})$ over $X$ assigned to a non-deterministic automaton is the set of elements of $X^{*}$ obtainable from the labels of all possible paths of arrows with source in $S_{0}$ and target in $F_{0}$; in this case we say that $L=L(\mathcal{A})$ is a language accepted by $\mathcal{A}$. We say $w$ is readable in $\mathcal{A}$ from $s_{1}$ to $s_{2}$, where $s_{1}, s_{2} \in S(\mathcal{A})$, if there is a path of arrows from $s_{1}$ to $s_{2}$ with the label $w$.

A deterministic finite state automaton can be considered a special case of a non-deterministic finite state automaton, for which the following conditions are satisfied: there are no arrows labelled $\varepsilon$; each state is the source of exactly one arrow with any given label from $X$; and $S_{0}$ has exactly one element.

By the Kleene-Rabin-Scott theorem a regular language over $X$ may be identified with the language accepted by some non-deterministic finite state automaton, or, equivalently, by some deterministic finite state automaton. If $R=L(\mathcal{A})$ is a subset of a group $F$, then $X$ is supposed to be a set of group generators for $F$, i.e. elements of $R$ are meant to be reduced strings over $X \cup X^{-1}$.

\subsection{Graphs associated with a subgroup and Schreier transver- sals}

We shall sometimes identify an automaton $\mathcal{A}$ with a finite connected oriented labelled graph $\Gamma$ with a distinguished vertex in natural way. Namely, 
take vertex set $V=V(\Gamma)=S(\mathcal{A})$; edge set $E=E(\Gamma)$ corresponding to the arrows, with induced labelling, and form subsets $S_{0}, F_{0} \subseteq S(\mathcal{A})$ from distinguished vertices. We shall also ascribe properties of automata to graphs.

One can associate with a subgroup $C$ two graphs: the subgroup graph $\Gamma=\Gamma_{C}$ and the Schreier graph $\Gamma^{*}=\Gamma_{C}^{*}$; we refer reader to 12] for more details on this subject.

Recall, that $\Gamma$ is a finite connected digraph with edges labelled by elements from $X$ and a distinguished vertex (based-point) 1 (so $S_{0}=F_{0}=\{1\}$ ), satisfying the following two conditions. Firstly, $\Gamma$ is folded, i.e., there are no two edges in $\Gamma$ with the same label and having the same initial or terminal vertices. Secondly, $\Gamma$ accepts precisely the reduced words in $X \cup X^{-1}$ that belong to $C$. To explain the latter observe, that walking along a path $p$ in $\Gamma$ one can read a word $\ell(p)$ in the alphabet $X \cup X^{-1}$, the label of $p$, (reading $x$ in passing an edge $e$ with label $x$ along the orientation of $e$, and reading $x^{-1}$ in the opposite direction). We say that $\Gamma$ accepts a word $w$ if $w=\ell(p)$ for some closed path $p$ that starts at 1 and has no backtracking. Clearly, $\Gamma$ can be identified with a deterministic finite state automata with 1 as the unique initial and final state.

The Schreier graph $\Gamma^{*}=\Gamma_{C}^{*}$ of $C$ is a connected labelled digraph with the set $\{C u \mid u \in F\}$ of the right cosets of $C$ in $F$ as the vertex set, and such that there is an edge from $C u$ to $C v$ with a label $x \in X$ if and only if $C u x=C v$. One can describe the Schreier graph $\Gamma^{*}$ as obtained from $\Gamma$ by the following procedure. Let $v \in \Gamma$ and $x \in X$ such that there is no outgoing or incoming edge at $v$ labelled by $x$. For every such $v$ and $x \in X$ we attach to $v$ a new edge $e$ (correspondingly, either outgoing or incoming) labelled $x$ with a new terminal vertex $u$ (not in $\Gamma$ ). Then we attach to $u$ the Cayley graph $C(F, X)$ of $F$ relative to $X$ (identifying $u$ with the vertex 1 of $C(F, X)$ ), and then we fold the edge $e$ with the corresponding edge in $C(F, X)$ (that is labelled $x$ and is incoming to $u$ ). Observe, that for every vertex $v \in \Gamma^{*}$ and every reduced word $w$ in $X \cup X^{-1}$ there is a unique path $\Gamma^{*}$ that starts at $v$ and has the label $w$. By $p_{w}$ we denote such a path that starts at 1 , and by $v_{w}$ the end vertex of $p_{w}$. The resulting graph $\Gamma_{C}^{*}$ is the Schreier graph of $C$ in $F$.

Notice that $\Gamma=\Gamma^{*}$ if and only if the subgroup $C$ has finite index in $F$. A spanning subtree $T$ of $\Gamma$ with the root at the vertex 1 is called geodesic if for every vertex $v \in V(\Gamma)$ the unique path in $T$ from 1 to $v$ is a geodesic path in $\Gamma$. For a given graph $\Gamma$ one can effectively construct a geodesic spanning subtree $T$.

From now on we fix an arbitrary spanning subtree $T$ of $\Gamma$. It is easy to see that the tree $T$ uniquely extends to a spanning subtree $T^{*}$ of $\Gamma^{*}$. 
Let $V\left(\Gamma^{*}\right)$ be the set of vertices of $\Gamma^{*}$. Since, in general, $\Gamma^{*}$ is infinite, we need to extend terminology from subsection 2.1. For a subset $Y \subseteq V\left(\Gamma^{*}\right)$ and a subgraph $\Delta$ of $\Gamma^{*}$, we define the language accepted by $\Delta$ and $Y$ as the set $L(\Delta, Y, 1)$ of the labels $\ell(p)$ of paths $p$ in $\Delta$ that start at 1 and end at one of the vertices in $Y$, and have no backtracking. Notice that the words $\ell(p)$ are reduced since the graph $\Gamma^{*}$ is folded. Notice, that $F=L\left(\Gamma^{*}, V\left(\Gamma^{*}\right), 1\right)$ and $C=L(\Gamma,\{1\}, 1)=L\left(\Gamma^{*},\{1\}, 1\right)$.

Sometimes we shall refer to a set of right representatives of $C$ as the transversal of $C$. Recall, that a transversal $S$ of $C$ is termed Schreier if every initial segment of a representative from $S$ belongs to $S$.

Further, let $S$ be a transversal of $C$. A representative $s \in S$ is called internal if the path $p_{s}$ ends in $\Gamma$, i.e., $v_{s} \in V(\Gamma)$. By $S_{\text {int }}$ we denote the set of all internal representatives in $S$. It follows from definition, that $\left|S_{\text {int }}\right|=$ $|V(\Gamma)|$.

A representative $s \in S$ is called geodesic if it has minimal possible length in its coset $C s$. The transversal $S$ is geodesic if every $s \in S$ is geodesic. Clearly, if $T^{*}$ is a geodesic subtree of $\Gamma^{*}$ (and hence $T$ is a geodesic subtree of $\Gamma$ ), then $S$ is a geodesic transversal.

\section{Main results on Double Cosets}

In this section we study double cosets from different points of view.

\subsection{Basic definitions and properties}

Let $F=F(X), C$ as above. Fix an element $f \in F \backslash C$ and consider the double coset $C f C$ for this element. Clearly, the following conditions are equivalent:

$$
\begin{aligned}
g \in C f C & \Leftrightarrow \exists d_{1}, d_{2} \in C \quad g=d_{1} f d_{2} \\
& \Leftrightarrow \exists d_{1}, d_{2} \in C \quad d_{1} g=f d_{2} .
\end{aligned}
$$

Denote by $E(g, f)$ the equation $x g=f y$ over $C$. Due to the latter equivalence, an element $g$ belongs to $C f C$ iff the equation $E(g, f)$ is solvable over $C$, i.e. exist $c_{1}, c_{2} \in C$ such that $x=c_{1}, y=c_{2}$. Lemma 3.1 describes some properties of solutions of the uniform equation $E(f, f)$.

We assign to $f$ a subgroup $C_{f} \subseteq F$ such that $C_{f}=C \cap C^{f}$, where $C^{f}$ is the set of all elements of the form $f^{-1} C f$. It turns out that $C_{f}$ plays a 
special role in the whole theory of double cosets. This subgroup is one of the most important characteristics of the embedding of $C$ in $F$.

Further, a subgroup $C$ is called $f$-malnormal if $C_{f}=1$. Recall that a subgroup $C$ is said to be malnormal if it is $f$-malnormal for all $f \in F \backslash C$. If, on the contrary, $C_{f}$ is nontrivial then, following [1], we call $f$ potentially normalizing (or pn for short).

Lemma 3.1. Let $C<F$ and $f \in F \backslash C$. Let $D(f, f) \subseteq C \times C$ be the set of all solutions of equation $E(f, f)$. In the notation above

- $D(f, f)$ consists of pairs $\left(c, c^{f^{-1}}\right)$ for all $c \in C_{f}$;

- if $C$ is $f$-malnormal (malnormal a fortiori), then $\sharp D(f, f)=1$;

- if $g \in C f C$, then $C_{g}$ is isomorphic to $C_{f}$.

Proof. To show (1) take an arbitrary $c_{1} \in C_{f}$; then by definition $c_{1} \in$ $C^{f} \cap C$. Therefore, exists an element $c_{2}$ such that $c_{1}=f^{-1} c_{2} f$. Evidently, $\left(c_{1}, c_{1}^{f^{-1}}\right)$ provide a solution of $E(f, f)$ and all such elements can be obtained analogously.

Let us show (2). Suppose there exist two different solutions of $E(f, f)$, say $c_{1}, c_{2}$ and $d_{1}, d_{2}$ in $C \backslash\{1\}$. Then $c_{1}=c_{2}^{f^{-1}}, d_{1}=d_{2}^{f^{-1}}$ and therefore $\left(c_{1} d_{1}^{-1}\right)^{f}=c_{2} d_{2}^{-1}$. From this it follows that $c_{1} d_{1}^{-1}, c_{2} d_{2}^{-1} \in C_{f}=1$. Thus $c_{1}=d_{1}$ and $c_{2}=d_{2}$ and the set $D(f, f)$ consists of only one pair.

To prove claim (3), suppose $g=d_{1} f d_{2}$ for some $d_{1}, d_{2} \in C$. By definition $C_{g}=C^{g} \cap C=C^{d_{1} f d_{2}} \cap C=\left(C^{d_{1}}\right)^{f d_{2}} \cap C \simeq C^{f} \cap C^{d_{2}^{-1}}=C^{f} \cap C=C_{f}$. This concludes the proof.

Remark 3.2. If an element $f$ is pn, then every other representative in $C f C$ is also potentially normalizing by Lemma 3.1 .

A double coset $C f C$ is called essential if $C_{f} \neq 1$ for some representative $f \in F$ (and so for all representatives of this coset).

Lemma 3.3. The number of different essential cosets is finite.

Proof. The union of all essential double cosets forms so called generalized normalizer of $C$ in $F$, i.e. the set $N_{F}^{*}(C)=\left\{f \in F \mid f^{-1} C f \cap C \neq 1\right\}$. This union is known to be finite (see, for example, [8], proposition 3.5.). 
Lemma 3.4. Let $C<F$ and $f, g \in F \backslash C$. If the equation $E(g, f)$ has a solution $\left(c_{1}, c_{2}\right)$ over $C$, then the set $D(g, f)$ of all solutions of this equation consist of all pairs $\left(c_{f} c_{1}, c_{2} c_{f}^{f c_{2}}\right)$, where $c_{f} \in C_{f^{-1}}$. In particular, if $C$ is $f$-malnormal, then there exists a unique solution $\left(c_{1}, c_{2}\right)$ with $c_{1}, c_{2} \in C$ such that $g=c_{1} f c_{2}$ for $g \in C f C$.

Proof. Notice that $E(g, f)$ is consistent by assumption and therefore exists a pair $\left(c_{1}, c_{2}\right)$ such that $c_{1} g=f c_{2}$. If $C$ is $f$-malnormal, then $C_{f} \simeq C_{g}=1$ and $D(g, f)$ satisfies the desired conclusion. Denote by $M$ the set of all such pairs and suppose there exists another pair of solutions, say $\left(d_{1}, d_{2}\right)$. Then $g^{-1} d_{1}^{-1} c_{1} g=d_{2}^{-1} c_{2}$ and $f d_{2} c_{2}^{-1} f^{-1}=d_{1} c_{1}^{-1}$, and therefore $d_{2}^{-1} c_{2}=c_{g} \in C_{g}$ and $d_{1} c_{1}^{-1}=c_{f} \in C_{f^{-1}}$. This implies $d_{1}=c_{f} c_{1}$ and $d_{2}=c_{2} c_{g}^{-1}$, but $c_{g}^{-1}=c_{f}^{c_{1} g}=c_{f}^{f c_{2}}$ and $\left(d_{1}, d_{2}\right)=\left(c_{f} c_{1}, c_{2} c_{f}^{f c_{2}}\right) \in M$. The opposite inclusion $M \subseteq D(g, f)$ is straightforward.

\subsection{Advantages of Nielsen set of generators}

Now suppose that $C$ is supplied by a Nielsen generating set. In this subsection we analyze how this affects the cancellation in cosets and double cosets of $C$. We also introduce here the notion of a relative Schreier transversal of $C$ with respect to its subgroup $D$.

Since an arbitrary set of generators for $C$ can be carried into a Nielsen set (see, for example, [12] or [15]), without loss of generality it can be assumed that $C$ is presented by such a set $Y=\left\{h_{1}, \ldots, h_{r}\right\}$. Let $S$ be the geodesic Schreier transversal for $C$ in $F$ such that the Nielsen set of generators $Y$ has the following properties with respect to $S$.

\section{Properties of a Nielsen set of generators:}

(i) each $h \in Y \cup Y^{-1}$ can be written in the form

$$
h=s_{1} \mu(h) s_{2}^{-1},
$$

where $s_{1}, s_{2} \in S_{\text {int }}$ are written as reduced $X$-words, $\mu(h)$ is an element of $X \cup X^{-1}$ and

$$
\left|l_{X}\left(s_{1}\right)-l_{X}\left(s_{2}\right)\right| \leq 1 ;
$$

(ii) if $h_{i_{1}}, h_{i_{2}}$ are distinct elements of $Y$, then the letters $\mu\left(h_{i_{1}}\right)^{ \pm 1}$ and $\mu\left(h_{i_{2}}\right)^{ \pm 1}$ do not cancel on computing the $X$-reduced form of $h_{i_{1}}^{ \pm 1} h_{i_{2}}^{ \pm 1}$;

(iii) if $h_{i_{1}}, h_{i_{2}}, h_{i_{3}}$ are elements of $Y \cup Y^{-1}$ such that $h_{i_{2}} \neq h_{i_{1}}^{-1}$ and $h_{i_{2}} \neq h_{i_{3}}^{-1}$, then the letter $\mu\left(h_{i_{2}}\right)$ does not cancel on computing the $X$-reduced form of $h_{i_{1}} h_{i_{2}} h_{i_{3}}$. 
Clearly, such $S$ always exists; and the letter $\mu(h)$ is called the central letter of $h \in Y \cup Y^{-1}$. Set $M=M(Y)=\left[\frac{1}{2} \max \left\{l_{X}\left(h_{1}\right), \ldots, l_{X}\left(h_{r}\right)\right\}\right]+1$.

The following material (up to Lemma [3.7) can be extracted from [1].

Lemma 3.5. [1, Lemma 2] Suppose $f \in F$ is pn and has minimal length in $C f C$. If $f c_{1} f^{-1}=c_{2}$, where $c_{1}, c_{2} \in C, c_{1} \neq 1$, then at least one of the letters of $c_{1}$ appears in the reduced form of $f c_{1} f^{-1}$, i.e., in $c_{2}$.

Lemma 3.6. [1, Lemma 3,4] Suppose $f$ is of minimal length in $f C$ and that $c=h_{i_{1}} \ldots h_{i_{n}}$ (where $\left.h_{i_{1}}, \ldots, h_{i_{n}} \in Y \cup Y^{-1}\right)$ is $Y$-reduced.

Then either $\mu\left(h_{i_{1}}\right)$ remains in $f h_{i_{1}} \ldots h_{i_{n}}$ and in this case the cancellation with $f$ in $f c$ is exactly that of $f$ in $f h_{i_{1}}$; or the central letter $\mu\left(h_{i_{1}}\right)$ cancels in the product $f c$, and in this case $h_{i_{1}}$ is of even length and exactly half of $h_{i_{1}}$ cancels with $f$;

Suppose that $\mu\left(h_{i_{j}}\right)$ cancels in the product $f h_{i_{1}} \ldots h_{i_{j}} \ldots h_{i_{n}}$, but $\mu\left(h_{i_{j+1}}\right)$ does not. Then

$j \leq r ;$

for any $k=1, \ldots, j$ the length $l_{X}\left(h_{i_{k}}\right)$ is even and $\mu\left(h_{i_{k}}\right)$ cancels in product $f c$;

for any $k=1, \ldots, j-1$ exactly the right half $s_{2 k}^{-1}$ of $h_{i_{k}}$ cancels completely in $h_{i_{k}} h_{i_{k+1}}$;

$l_{X}\left(h_{i_{1}}\right)<\ldots<l_{X}\left(h_{i_{j}}\right)$;

if the right half of $h_{i_{j}}$ does not cancel completely with $h_{i_{j+1}}$, then $h_{i_{1}} \ldots h_{i_{j-1}} s_{1 j} \mu\left(h_{i_{j}}\right)$ is precisely the part of $c$ that cancels with $f$ and $l_{X}\left(h_{i_{1}} \ldots h_{i_{j-1}} s_{1 j} \mu\left(h_{i_{j}}\right)\right)=\frac{1}{2} l_{X}\left(h_{i_{j}}\right)$;

if the right half of $h_{i_{j}}$ does cancel with $h_{i_{j+1}}$, then $h_{i_{1}} \ldots h_{i_{j}} s$ is precisely the part of $c$ that cancels with $f$ for some $s \in S_{\text {int }}$ and $l_{X}\left(h_{i_{1}} \ldots h_{i_{j}} s\right) \leq \frac{1}{2} l_{X}\left(h_{i_{j+1}}\right)$.

Lemma 3.7. [1, Lemma 5] Suppose that the equation $E(f, f)$ is satisfied for $c_{1}, c_{2} \in C$, and $f$ is of minimal length in $C f C$. Then there exists $f^{\prime} \in C f C$ of the same length as $f$ which is a product of two pieces of generators from $Y \cup Y^{-1}$.

Lemma 3.7 allows us to obtain an algorithm which lists all essential double cosets: 
Corollary 3.8. There exists an algorithm $A$, which given a subgroup $C$ of $F$ and a Nielsen set of generators $Y=\left\{h_{1}, \ldots, h_{r}\right\}$ lists all essential double cosets $C f C$ of $F$ (finite in their number by Lemma 3.3). Moreover, $A$ runs in polynomial time in $M=M(Y)=\left[\frac{1}{2} \max \left\{l_{X}\left(h_{1}\right), \ldots, l_{X}\left(h_{r}\right)\right\}\right]+1$.

Proof. The number of different pieces of generators in $Y \cup Y^{-1}$ can be estimated as $O\left(M^{2}\right)$, and therefore one can form a list of all possible products $f^{\prime} \in C f C$ (as in 3.7) in time at most $O\left(M^{4}\right)$. Let us fix an element $f \in F \backslash C$ of this list. By [17, Theorem 1.6], we can construct subgroup graphs $\Gamma_{C_{f}}$ and $\Gamma_{C}$ in time $\Theta(N+4 M)$ and $\Theta(N)$, respectively, where $N \leq 2 M r$ and $\Theta(x)=O\left(x \log ^{*}(x)\right)$. Here the function $\log ^{*}: \mathbb{N} \rightarrow \mathbb{N}$ is given by $\log ^{*}(x)=k$, where $k=\min \left\{m \in \mathbb{N}: \log ^{m}(x) \leq 1\right\}$. The product $\Gamma_{C_{f}} \times \Gamma_{C}$ can be constructed in time $O((N+4 M) N)$, which is bounded above by $O\left(M^{2}\right)$. If now the component of $\Gamma_{C_{f}} \times \Gamma_{C}$ containing $1_{C_{f}} \times 1_{C}$ is not an isolated vertex, then $f$ is essential.

Using properties of sets of Nielsen generators and Lemmas 3.5, 3.6, we want to introduce a notion of the relative Schreier transversal. We shall use this to simplify multiplication and representation of elements in double cosets.

For every pair $h_{i}, h_{j} \in Y^{ \pm 1}, h_{i} \neq h_{j}^{-1}$, define $a_{i j}$ to be the initial subword of $h_{i}$ that does not cancel on forming the product $h_{i} h_{j}$, and let $b_{i j}$ be the terminal subword of $h_{j}$ that does not cancel on forming $h_{i} h_{j}$.

Denote also $m_{i j k}$ the subword of $h_{j}$ that does not cancel on forming the triple product $h_{i} h_{j} h_{k}$, where $h_{j} \neq h_{k}^{-1}, h_{j} \neq h_{i}^{-1}$. Notice that by definition of a Nielsen set of generators all words $a_{i j}, b_{i j}$ and $m_{i j k}$ in $F(X)$ are nontrivial. Denote by $\Sigma$ this new alphabet $\left\{a_{i j}\right\} \cup\left\{b_{i j}\right\} \cup\left\{m_{i j k}\right\} \cup\left\{h_{i}\right\}$ obtained from all such generators, their pairs $h_{i}, h_{j}$ and triples $h_{i}, h_{j}, h_{k}$. We also use an additional subdivision of $m_{i j k}$. Namely, let $m_{i j k}=\alpha_{i j} \circ \mu_{j} \circ \beta_{j k}$ for some $\alpha_{i j}, \beta_{j k} \in F(X)$ (not necessarily non-trivial). (In Section 3.3 we subdivide $\alpha$-s and $\beta$ 's into smaller pieces: $\left.m_{i j k}=\alpha_{1 i j} \circ \alpha_{2 i j} \circ \mu_{j} \circ \beta_{2 j k} \circ \beta_{1 j k}\right)$.

A nontrivial $\Sigma$-reduced word $u$ is called $C$-admissible if it has one of the following forms

$$
\begin{gathered}
c=a_{i_{1} i_{2}} \circ m_{i_{1} i_{2} i_{3}} \circ \ldots \circ m_{i_{k-2} i_{k-1} i_{k}} \circ b_{i_{k-1} i_{k}} \text { or } \\
c=a_{i_{1} i_{2}} \circ b_{i_{1} i_{2}} \text { or } c=h_{i} .
\end{gathered}
$$

Clearly, there is one-to-one correspondence between $C$-admissible words and nontrivial $X$-reduced words $c \in C$.

Further, let $D$ be a nontrivial subgroup of $C$ and suppose $D$ is given by a finite Nielsen set of generators $Z=\left\{d_{1}, \ldots, d_{m}\right\}$, where $d_{1}, \ldots, d_{m}$ are 
$Y$-reduced words. Consider a subgroup graph $\Gamma_{D}$, and take a maximal subtree $\Upsilon$ of $\Gamma_{D}$ such that the corresponding Schreier transversal $T$ respect the choice of central letters $\mu\left(d_{i}\right)$. Denote by $T_{\text {int }}$ the set of all inner representatives from $T$. Combining properties (i), (ii), (iii) of a Nielsen set of generators with formulae (11) - (2) above, we get

$$
\begin{aligned}
d_{i} & =t_{1} \cdot \mu\left(d_{i}\right) \cdot t_{2}^{-1} \\
& =\left(a_{i_{1} i_{2}} \circ m_{i_{1} i_{2} i_{3}} \circ \ldots\right) \circ m_{i_{j-1} i_{j} i_{j+1}} \circ\left(\ldots \circ m_{i_{k} i_{k+1} i_{k+2}} \circ b_{i_{k+1} i_{k+2}}\right) \text { or } \\
d_{i} & =a_{i_{1} i_{2}} \circ m_{i_{1} i_{2} i_{3}} \circ b_{i_{2} i_{3}} \text { or } \\
d_{i} & =a_{i_{1} i_{2}} \circ b_{i_{1} i_{2}} \text { or } d_{i}=h_{i_{1}},
\end{aligned}
$$

where $t_{1}, t_{2} \in T_{\text {int }} ; \mu\left(d_{i}\right), h_{i_{1}} \in Y \cup Y^{-1}$, and $a_{i j}, b_{i j}, m_{i j k} \in \Sigma$.

The system $T_{D}$ of all $C$-admissible words $t \in T$ is called the relative Schreier transversal of $C$ in $F$ with respect to $D$ and $\Upsilon$.

Example 3.9. Consider an example of a relative Schreier transversal for a subgroup $C<F(a, b)$. Let $Y=\left\{a^{3}, b^{3}, a b^{2} a^{-1}, b a^{3} b^{-1}, b a b^{2} a^{-1} b^{-1}\right\}=$ $\left\{h_{1}, h_{2}, h_{3}, h_{4}, h_{5}\right\}$ be a Nielsen set of generators of $C$. The subgroup graph $\Gamma_{C}$ is shown on figure1, the edges entering maximal subtree are highlighted.

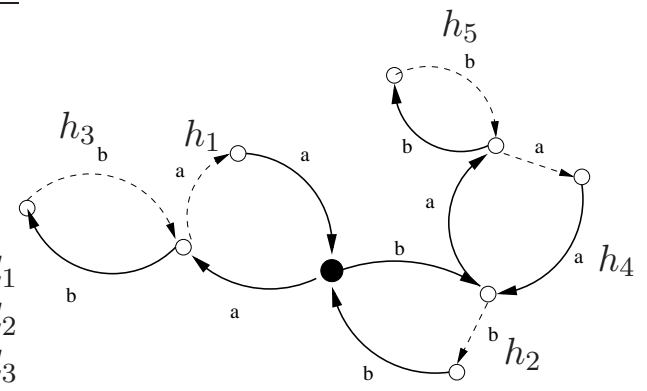

Figure 1: Stallings automaton $\Gamma_{C}$ recognizing $C=$ $\left\langle a^{3}, b^{3}, a b^{2} a^{-1}, b a^{3} b^{-1}, b a b^{2} a^{-1} b^{-1}\right\rangle$

Further, represent elements of $Y \cup Y^{-1}$ in the form $h_{i}=s_{1 i} \circ \mu_{i} \circ s_{2 i}^{-1}$ (uniquely determined by $S$ ):

$$
\begin{gathered}
h_{1}=a \circ a \circ a=h_{6}^{-1}, \quad h_{2}=b \circ b \circ b=h_{7}^{-1}, \\
h_{3}=a b \circ b \circ a^{-1}=h_{8}^{-1}, \quad h_{4}=b a \circ a \circ a b^{-1}=h_{9}^{-1}, \\
h_{5}=b a b \circ b \circ a^{-1} b^{-1}=h_{10}^{-1} .
\end{gathered}
$$


Taking all suitable products of $h_{i}, h_{j}$ and $h_{k}$ for $i, j, k=1, \ldots, 10$, one can easily construct $\Sigma$. For instance, $a_{11}=a^{3}, a_{74}=b^{-2}, m_{123}=b^{3}, m_{742}=a^{3}$, $b_{42}=b^{2}$ etc. The element $a$ is pn and a subgroup $C_{a}$ is generated by a Nielsen generating set $Z=\left\{h_{2}^{-1} h_{4} h_{2}, h_{1}, h_{2}^{2}\right\}=\left\{d_{1}, d_{2}, d_{3}\right\}$. Then, taking a maximal subtree $\Upsilon$ as shown on figure 2(a), obtain

$$
d_{1}=a_{74} \circ m_{742} \circ b_{42}, \quad d_{2}=h_{1} \quad \text { and } \quad d_{3}=a_{22} \circ b_{22} .
$$

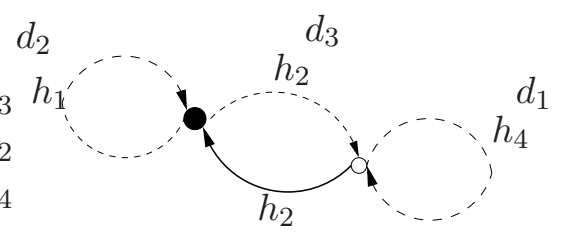

(a) Subgroup graph $\Gamma_{D}$

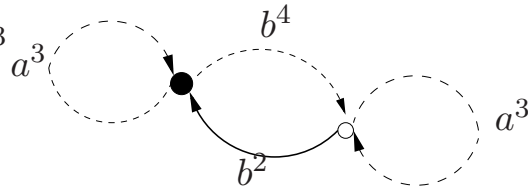

(b) Consolidated graph $\Gamma_{D}^{\prime}$

Figure 2: Subgroup graph $\Gamma_{D}$ and consolidated graph $\Gamma_{D}^{\prime}$ for $D=$ $\left\langle h_{2}^{-1} h_{4} h_{2}, h_{1}, h_{2}^{2}\right\rangle$

Therefore, $m_{742}, h_{1}$ and $a_{22}$ are central words and $1, a_{74}, b_{42}^{-1}, b_{22}$ forms the inner part of the relative Schreier transversal for $C$ in $F$ with respect to $C_{a}$ and $\Upsilon$. This relative transversal in terms of $X=\{a, b\}$ is shown on figure 2(b) (and we refer reader to [4 for more details on consolidated graphs).

In the sequel, we omit a maximal subtree $\Upsilon$ from the notation assuming it fixed for every relative Schreier transversal.

In Lemma 3.4 we showed that every representative $g \in C f C$ has a unique presentation in the form $g=c_{1} f c_{2}, c_{1}, c_{2} \in C$ when $C_{f}=1$. However, this is not the case for essential cosets and such a presentation is not unique in general.

Nevertheless, the representatives of essential cosets can be uniquely written in a similar way:

Lemma 3.10. Let $C f C$ be an essential coset and let $T$ be a Schreier transversal for $C$ in $F$ with respect to $C_{f}$. Then every $g \in C f C$ has a unique presentation

$$
g=c f t, \quad c_{1} \in C, t \in T \text {. }
$$

Proof. Let $g=c_{1} f d_{1}$ be a representative of a some essential double coset $C f C, c_{1}, d_{1} \in C$. By definition of a relative Schreier transversal there is an element $t_{1} \in T$ such that $g=c_{1} f t_{1}$. Now suppose that $g=c_{2} f t_{2}$ is 
another presentation for $g, c_{2} \in C, t_{2} \in T$. Then $c_{1} f t_{1}=c_{2} f t_{2}$ implies $c_{1}^{-1} c_{2}=f t_{1} t_{2}^{-1} f^{-1}$ and since $t_{1} t_{2}^{-1} \in C$ we have $c_{1}^{-1} c_{2} \in C_{f}$ and $t_{1} \in C_{f} t_{2}$. But $t_{1}, t_{2}$ are both representatives of $C_{f}$ in $C$ and since they are in the same coset, $t_{1}=t_{2}$ and hence $c_{1}=c_{2}$.

\subsection{Cancellations in complex $C f C$}

In this subsection we estimate the size of cancellations in a double coset depending on a subgroup $C_{f}$.

Let $Y=\left\{h_{1}, \ldots, h_{r}\right\}$ be some fixed set of Nielsen generators for $C$. Recall that $M=\left[\frac{1}{2} \max \left\{l_{X}\left(h_{1}\right), \ldots, l_{X}\left(h_{r}\right)\right\}\right]+1$ and set $p$ equal to the number of elements in the ball of radius $2 M$ in $F(X)$. We shall use this notation in what follows. The following technical lemma turns out to be crucial in the proof of Theorem 3.12 .

Lemma 3.11. Let $f_{0}, f_{1}=h_{i_{1}} f_{0} h_{j_{1}}, \ldots, f_{k}=h_{i_{k}} f_{k-1} h_{j_{k}}$ be a sequence of elements in $F(X)$ such that $h_{i_{1}}, h_{j_{1}}, \ldots, h_{i_{k}}, h_{j_{k}}$ are elements of $Y \cup Y^{-1}$; $h_{i_{k}} \ldots h_{i_{1}}$ and $h_{j_{1}} \ldots h_{j_{k}}$ are $Y$-reduced words, and $l_{X}\left(f_{l}\right) \leq 2 M$ for all $l=1, \ldots, k$. Then for all $k \geq p$ there exist $l, n \in\{1, \ldots, k\}$ and nontrivial $c_{l}, c_{n}, d_{l}, d_{n} \in C$ such that $l \neq n$, and $c_{l} \neq c_{n}, d_{l} \neq d_{n}$, and

$$
c_{l} f_{l} d_{l}=c_{n} f_{l} d_{n}=c_{n} f_{n} d_{n}=c_{l} f_{n} d_{l} .
$$

Proof. Fix a number $k$; notice that $M$ is at least 1 so $p \geq 4 m^{2}+1$ for a free group $F$ of rank $m$ and therefore $k \geq p \geq 17$. In particular, $k>0$. The number of all elements of length not greater then $2 M$ is equal to $p$ by definition, and so by Dirichlet's drawer principle there are at least two equal elements among $k+1>p$ elements of such length, say $f_{l}$ and $f_{n}$ with $l<n$. The statement of the lemma is obvious for $f_{l}=f_{n}=1$, so suppose $f_{l}, f_{n}$ are non-trivial. Since

$$
h_{i_{k}} \ldots h_{i_{1}} f_{0} h_{j_{1}} \ldots h_{j_{k}}=h_{i_{k}} \ldots h_{i_{l+1}} f_{l} h_{j_{l+1}} \ldots h_{j_{k}}=f_{k}
$$

one can take $c_{l}=h_{i_{k}} \ldots h_{i_{l}}, c_{n}=h_{i_{k}} \ldots h_{i_{n}}$ and $d_{l}=h_{j_{l}} \ldots h_{i_{k}}, d_{n}=$ $h_{j_{n}} \ldots h_{i_{k}}$. Clearly, $c_{l}, c_{n}, d_{l}, d_{n}$ are non-trivial (as they are $Y$-reduced products of non-trivial elements ) and $c_{l} \neq c_{n}, d_{l} \neq d_{n}$. Indeed, let, for instance, $c_{l}=c_{n}$; then the product $c_{l}^{-1} c_{n}$ is equal to $h_{i l+1} \ldots h_{i_{k}}$. But $h_{i l+1} \ldots h_{i_{k}}$ can not be trivial as a $Y$-reduced word in Nielsen generators. Therefore, $c_{l} f_{l} d_{l}=c_{n} f_{l} d_{n}$ and other equalities are straightforward.

Let $A_{1}, A_{2}, \ldots, A_{n}$ be a finite number of subsets in $F(X)$ and consider the function $\mathrm{cn}: A_{1} \cdot A_{2} \cdot \ldots \cdot A_{n} \mapsto \mathbb{N}$ which computes the total amount of $X$-cancellations in the product $a_{1} \cdot \ldots \cdot a_{n}$ : 


$$
\operatorname{cn}\left(a_{1}, a_{2}, \ldots, a_{n}\right)=\frac{1}{2}\left(\sum_{i=1}^{n} l_{X}\left(a_{i}\right)-l_{X}\left(a_{1} a_{2} \cdot \ldots \cdot a_{n}\right)\right) .
$$

Observe that for $n=2$ the function $c n\left(a_{1}, a_{2}\right)$ coincides with the notion of Lyndon-Chiswell-Gromov product $\left(a_{1}, a_{2}^{-1}\right)$ in a group $F$ with respect to canonical length function $l_{X}$ (see [14, 6] for details on length functions, and in [10] this definition was adapted to hyperbolic metric spaces). We say that the product $A_{1} \cdot A_{2} \cdot \ldots \cdot A_{n}$ is $k$-reduced if there is a constant $k \geq 0 \mathrm{such}$ that for every $a_{i} \in A_{i}, i=1, \ldots, n$ the following holds

$$
\operatorname{cn}\left(a_{1}, a_{2}, \ldots, a_{n}\right) \leq k \text {. }
$$

For instance, two singletons $A_{1}=\{u\}, A_{2}=\{v\}$ such that $u v=u \circ v$ form a 0 -reduced set, but we shall omit the prefix 0 - below (saving " $k$-" only for $k>0)$.

Theorem 3.12. Let $F$ be a free group with a finite basis $X$ and $C$ be a finitely generated subgroup of $F$ with a Nielsen set of generators $Y$. Suppose $f \in F \backslash C$ and $f$ is of minimal length in a double coset $C f C$, and let $k=2 p M$.

- If $C$ is $f$-malnormal, then $C f C$ is $k$-reduced.

- If CfC is essential, then CfT is $k$-reduced, where $T$ is the relative Schreier transversal of $C$ in $F$ relative to $C_{f}$.

Proof. Let $g \in C f C$. If $f$ does not cancel completely in $g=c f d$, then by Lemma 3.6 the length of cancellations $c n(c, f)$ is bounded above by $M$ (and also $c n(f, d) \leq M)$. Hence $c n(c, f, d)=c n(c, f)+c n(f, d) \leq 2 M$ and both (1.) and (2.) follows. If, on the other hand, $f$ cancels completely then there are the two cases $C_{f}=1$ and $C_{f} \neq 1$ to be considered.

1. Suppose $C_{f}=1$ and $g \in C f C$ such that the total amount of cancellations in $c f d=g$ is greater than $k$ for $c=h_{i_{1}} \ldots h_{i_{k}}, d=h_{j_{1}} \ldots h_{j_{n}} \in C$.

Let $f=f^{\prime} \circ f^{\prime \prime}$, where $f^{\prime}$ cancels completely in $c f$ and $f^{\prime \prime}$ cancels in $f d$. Below we list all possible forms of pieces $f^{\prime}$ and $f^{\prime \prime}$. By Lemma 3.6 $f^{\prime \prime}$ possesses the following properties:

1.1. either $\left(f^{\prime \prime}\right)^{-1}=s$, where $s \in S_{\text {int }}$ and $s$ is an initial segment of $s_{1 j_{1}}$. Then $f^{\prime \prime} d=\gamma h_{j_{2}} \cdots h_{j_{n}}$ and $\gamma^{-1}$ is an initial segment of $h_{j_{1}}^{-1}$ of length $l_{X}(\gamma)<l_{X}\left(h_{j_{1}}\right) \leq 2 M$, and $l_{X}\left(f^{\prime \prime}\right) \leq M$; 
1.2. or $\left(f^{\prime \prime}\right)^{-1}=s_{1 j_{1}} \mu_{j_{1}}$. Then $f^{\prime \prime} d=s_{2 j_{1}}^{-1} h_{j_{2}} \cdots h_{j_{n}}$ and $l_{X}\left(s_{2 j_{1}}^{-1}\right) \leq M$, $l_{X}\left(f^{\prime \prime}\right) \leq M$

1.3. or $\left(f^{\prime \prime}\right)^{-1}=a_{j_{1} j_{2}} \circ m_{j_{1} j_{2} j_{3}} \circ m_{j_{2} j_{3} j_{4}} \circ \cdots \circ \circ m_{j_{s-2} j_{s-1} j_{s}} \circ \alpha \circ \mu_{j_{s}}$, in which case $f^{\prime \prime} d=\beta h_{j_{s+1}} \cdots h_{j_{n}}$; recall that $\alpha, \beta$ are elements of $F(X)$ such that $m_{j_{s-1} j_{s} j_{s+1}}=\alpha \circ \mu_{j_{s}} \circ \beta$. Here the length $l_{X}\left(f^{\prime \prime}\right) \leq M$ (see Lemma 3.6. (e)) and $l_{X}(\beta) \leq M$;

1.4. or $\left(f^{\prime \prime}\right)^{-1}=a_{j_{1} j_{2}} \circ m_{j_{1} j_{2} j_{3}} \circ m_{j_{2} j_{3} j_{4}} \circ \cdots \circ \circ m_{j_{s-2} j_{s-1} j_{s}} \circ \alpha_{1}$, where $f^{\prime \prime} d=\alpha_{2} \mu_{j_{s}} \beta h_{j_{s+1}} \cdots h_{j_{n}}$; and $\alpha_{1}, \alpha_{2}, \beta$ are such that $m_{j_{s-1} j_{s} j_{s+1}}=$ $\alpha_{1} \alpha_{2} \circ \mu_{j_{s}} \circ \beta$. Here the length $l_{X}\left(f^{\prime \prime}\right) \leq M$ (see (f) of Lemma 3.6) and $l_{X}\left(\alpha_{2} \mu_{j_{s}} \beta\right) \leq l_{X}\left(h_{j_{s}}\right) \leq 2 M$.

Similarly, $f^{\prime}$ and $c f^{\prime}$ have one of the forms:

2.1. either $\left(f^{\prime}\right)^{-1}=s$, where $s \in S_{\text {int }}$ and $s$ is a terminal segment of $s_{2 i_{k}}$. Then $c f^{\prime}=h_{i_{1}} \cdots h_{i_{k-1}} \gamma$ and $\gamma^{-1}$ is a beginning of $h_{i_{k}}$ of length $l_{X}(\gamma) \leq 2 M$, and $l_{X}\left(f^{\prime}\right) \leq M$

2.2 or $\left(f^{\prime}\right)^{-1}=\mu_{i_{k}} s_{2 i_{k}}^{-1}$. Then $c f^{\prime}=h_{i_{1}} \cdots h_{i_{k-1}} s_{1 i_{k}}$ and $l_{X}\left(s_{1 i_{k}}\right) \leq M$, $l_{X}\left(f^{\prime}\right) \leq M$

2.3. or $\left(f^{\prime}\right)^{-1}=\mu_{i_{l}} \circ \beta \circ m_{i_{l} i_{l+1} i_{l+2}} \circ m_{i_{l+1} i_{l+2} i_{l+3}} \circ \cdots \circ m_{i_{k-2} i_{k-1} i_{k}} \circ b_{i_{k-1} i_{k}}$, in which case $c f^{\prime}=h_{i_{1}} \cdots h_{i_{l-1}} \alpha$; here $\alpha, \beta$ are such that $m_{i_{l-1} i_{l} i_{l+1}}=$ $\alpha \circ \mu_{i_{l}} \circ \beta$. Here the length $l_{X}\left(f^{\prime}\right) \leq M$ and $l_{X}(\alpha) \leq M$;

2.4. or $\left(f^{\prime}\right)^{-1}=\beta_{2} m_{i_{l} i_{l+1} i_{l+2}} \circ m_{i_{l+1} i_{l+2} i_{l+3}} \circ \cdots \circ m_{i_{k-2} i_{k-1} i_{k}} \circ b_{i_{k-1} i_{k}}$, where $c f^{\prime}=h_{i_{1}} \cdots h_{i_{l-1}} \alpha \circ \mu_{i_{l}} \circ \beta_{1}$; and $\beta_{1}, \beta_{2}, \alpha$ are such that $m_{i_{l-1} i_{l} i_{l+1}}=$ $\alpha \circ \mu_{i_{l}} \circ \beta_{1} \beta_{2}$. Here the length $l_{X}\left(f^{\prime}\right) \leq M$ and $l_{X}\left(\alpha \mu_{i_{l}} \beta_{1}\right) \leq 2 M$.

Summarizing conditions $(1.1)-(2.4)$ and renumbering $h_{i_{1}}, \ldots, h_{j_{n}}$ for notational simplicity if necessary, we obtain

$$
g=h_{i_{1}} \cdots h_{i_{k}} f^{\prime} f^{\prime \prime} h_{j_{1}} \cdots h_{j_{n}}=h_{i_{1}} \cdots h_{i_{l-1}} q_{1} q_{2} h_{j_{s+1}} \cdots h_{j_{n}}
$$

where

$$
q_{1}=\left\{\begin{array}{r}
\gamma_{1}, \text { see }(2.1), \text { and } l_{X}\left(\gamma_{1}\right) \leq 2 M, \\
s_{1 i}, \text { see }(2.2-2.3), \text { and } l_{X}\left(s_{1 i}\right) \leq M, \\
s_{1 i} \mu_{i} \beta_{1}, \text { see }(2.4), \text { and } l_{X}\left(s_{1 i} \mu_{i} \beta_{1}\right) \leq 2 M
\end{array}\right.
$$


and

$$
q_{2}=\left\{\begin{array}{r}
\gamma_{2}, \text { see }(1.1), \text { and } l_{X}\left(\gamma_{2}\right) \leq 2 M, \\
s_{2 j}^{-1}, \text { see }(1.2-1.3), \text { and } l_{X}\left(s_{2 j}\right) \leq M, \\
\alpha_{2} \mu_{i} s_{2 j}^{-1}, \text { see }(1.4), \text { and } l_{X}\left(\alpha_{2} \mu_{i} s_{2 j}^{-1}\right) \leq 2 M .
\end{array}\right.
$$

If $q_{1} q_{2}=q_{1} \circ q_{2}$, then again $c n(c, f, d)=c n(c, f)+c n(f, d)$ and the total cancellations in $c f d$ are bounded above by $2 M$, in contradiction with assumption $c n(c, f, d)>k$. Therefore, $q_{1} q_{2}$ is not reduced. Notice that $f \notin C$ implies $g \notin C$ and therefore $q_{1} q_{2} \notin C$. Suppose $q_{1} q_{2}$ cancels to $q_{1}^{\prime} \circ q_{2}^{\prime}$, where $q_{1}^{\prime}$ is an initial part of $q_{1}$ and $q_{2}^{\prime}$ is a terminal part of $q_{2}$. Without loss of generality one can assume that all $h_{i_{1}}, \ldots, h_{j_{n}}$ cancel in

$$
c f d=h_{i_{1}} \cdots h_{i_{l-1}} q_{1}^{\prime} \cdot q_{2}^{\prime} h_{j_{s+1}} \cdots h_{j_{n}} .
$$

By definition of the function $c n$ we have

$$
c n(c, f, d)=\frac{1}{2}\left(l_{X}(c)+l_{X}(f)+l_{X}(d)-l_{X}(g)\right),
$$

where

$$
\begin{aligned}
& l_{X}(c)=\sum_{t=1}^{k} l_{X}\left(h_{i_{t}}\right)-2 \sum_{t=1}^{k-1} c n\left(h_{i_{t}}, h_{i_{t+1}}\right), \\
& l_{X}(d)=\sum_{z=1}^{n} l_{X}\left(h_{j_{z}}\right)-2 \sum_{z=1}^{n-1} c n\left(h_{j_{z}}, h_{j_{z+1}}\right),
\end{aligned}
$$

and $l_{X}(f)=l_{X}\left(f^{\prime}\right)+l_{X}\left(f^{\prime \prime}\right)$.

Rearranging summands in (44) and (3) we get

$$
\begin{gathered}
c n(c, f, d)=c n\left(h_{i_{l}} \cdot \ldots \cdot h_{i_{k}}, f^{\prime}\right)+c n\left(f^{\prime \prime}, h_{j_{1}} \cdot \ldots \cdot h_{j_{s}}\right)- \\
-\sum_{t=1}^{l-1} c n\left(h_{i_{t}}, h_{i_{t+1}}\right)-\sum_{z=s}^{n-1} c n\left(h_{j_{z}}, h_{j_{z+1}}\right)+ \\
+\frac{1}{2}\left(l_{X}\left(q_{2}\right)+l_{X}\left(q_{1}\right)+\sum_{t=1}^{l-1} l_{X}\left(h_{i_{t}}\right)+\sum_{z=s+1}^{n} l_{X}\left(h_{j_{z}}\right)-l_{X}(g)\right) .
\end{gathered}
$$

Further, formulae (1.1) - (2.4) imply $c n\left(h_{i_{l}} \cdot \ldots \cdot h_{i_{k}}, f^{\prime}\right) \leq M, c n\left(f^{\prime \prime}, h_{j_{1}}\right.$. $\left.\ldots \cdot h_{j_{s}}\right) \leq M$, and $l_{X}\left(q_{1}\right) \leq 2 M, l_{X}\left(q_{2}\right) \leq 2 M$. By definition of a Nielsen 
set of generators $\mathrm{cn}\left(h_{i}, h_{j}\right) \geq 0$ and $l_{X}\left(h_{i}\right) \leq 2 M$ for all $h_{i}, h_{j} \in Y \cup Y^{-1}$. Therefore,

$$
k<c n(c, f, d) \leq(l+n-s+3) M-\frac{1}{2} l_{X}(g) .
$$

Moreover, the assumption that all $h_{i_{1}}, \ldots, h_{j_{n}}$ cancel in (3) implies $l-1=$ $n-s$ and

$$
g=a_{i_{1} i_{2}}^{\prime} \circ b_{j_{1} j_{2}}^{\prime},
$$

where $a_{i_{1} i_{2}}^{\prime}$ is an initial segment of $a_{i_{1} i_{2}} \in \Sigma, b_{j_{1} j_{2}}^{\prime}$ is a terminal segment of $b_{j_{n-1} j_{n}} \in \Sigma$ and $g$ is not $C$-admissible. Hence, $\left(i_{1}, i_{2}\right) \neq\left(j_{n-1}, j_{n}\right)$ and since $l_{X}(g)>0$, we have

$$
0<l_{X}(g) \leq 4 M(l+1)-4 p M \Rightarrow l>p-1 .
$$

Consider the sequence

$$
\begin{aligned}
f_{0} & =f \\
f_{1} & =h_{i_{l}} \cdot \ldots \cdot h_{i_{k}} f_{0} h_{j_{1}} \cdot \ldots \cdot h_{j_{s}}, \\
f_{2} & =h_{i_{l-1}} f_{1} h_{j_{s+1}}, \\
& \ldots \\
f_{l-1} & =h_{i_{2}} f_{l-2} h_{j_{n-s}}, \\
f_{l} & =g
\end{aligned}
$$

Here $l_{X}\left(f_{0}\right)=l_{X}\left(f^{\prime}\right)+l_{X}\left(f^{\prime \prime}\right)$ and by $(1.1)$ - (2.4) we have $l_{X}\left(f_{0}\right) \leq 2 M$ and from formula (5) one can easily deduce $l_{X}\left(f_{l}\right) \leq 2 M$.

Further, since $f_{1}=q_{1}^{\prime} \circ q_{2}^{\prime}$, and $c, d$ are $Y$-reduced words in the Nielsen set of generators $Y$ (see properties (i) - (iii)), the equality (5) is possible only if all lengths $l_{X}\left(f_{1}\right), \ldots, l_{X}\left(f_{l-1}\right)$ are bounded above by $2 M$.

Observe that $l>p-1$ due to (6), and $f_{0}, \ldots, f_{l}$ represent the same double coset. Hence by Lemma 3.1. (3.) we have $C_{f}=C_{f_{0}} \simeq \ldots \simeq C_{f_{l}} \simeq$ $C_{f_{l+1}}=C_{g}$.

Therefore, $f_{0}, \ldots, f_{l}$ satisfy the assumptions of Lemma 3.11 and hence there exist elements, say $f_{i}, f_{j}, c_{i}, d_{i}, c_{j}$ and $d_{j}$ such that $c_{i} \neq c_{j}, d_{i} \neq d_{j}$, but $c_{i} f_{i} d_{i}=c_{j} f_{i} d_{j}$, a contradiction to the uniqueness of the representation of $f_{i}$ in $C f C$ (see Lemma 3.4).

2. Let $C_{f} \neq 1$. Then by Lemma 3.10 there is a unique presentation $g=c f d$ for $c \in C$ and $d \in T$ for the relative Schreier transversal $T=T_{C_{f}}$ 
(recall that we fixed a maximal subtree $\Upsilon$ in $\Gamma_{C_{f}}$; hence $T$ is unique). Then considering elements $d \in T$ and arguing as above, we obtain the equality $g=c_{i} f_{i} d_{i}=c_{j} f_{i} d_{j}$ for different indexes $i, j$ again, a contradiction with Lemma 3.10.

Remark 3.13. Notice that for essential double cosets the complex $C f C$ might not be $k$-reduced for any $k$. Indeed, let $w$ be a primitive element of an arbitrary finite rank free group $F$, and let $C$ be the subgroup of $F$ generated by $w^{l}$ for some $l>1$. Then $f=w$ is pn and does not belong to $C$. However, the total amount of cancellations in the product $w^{k l} w w^{-k l} \in C f C$ can be arbitrary large.

\subsection{Formal language and automatic properties}

In this subsection we investigate connections between bounded cancellation and regularity of subsets of $F(X)$. We establish the regularity of all reduced words representing elements of a double coset $C f C$ in a free group $F(X)$ using this relation.

Remark 3.14. Suppose $A_{1}, A_{2}$ are two regular subsets in $F$. Then the set $\overline{A_{1} A_{2}}$ of all reduced words in $A_{1} A_{2}$ is regular in $F(X)$.

The set $A_{1} A_{2}$ is regular by Benois' theorem (see [2]), but we also need an automaton accepting $\overline{A_{1} A_{2}}$. We shall show below how to construct this automaton for the case where the product $A_{1} A_{2}$ is $k$-reduced. Let $\mathcal{A}_{i}=$ $\left(S\left(\mathcal{A}_{i}\right), X, \delta_{i}, S_{0}\left(\mathcal{A}_{i}\right), F_{0}\left(\mathcal{A}_{i}\right)\right)$ be a deterministic automaton accepting $A_{i}$; in particular, it means $S_{0}\left(\mathcal{A}_{i}\right)=\left\{s_{0}\left(\mathcal{A}_{i}\right)\right\}, i=1,2$. One can easily form a (non-deterministic) automaton $\mathcal{A}$, which accepts the concatenation $A_{1} A_{2}$. Namely, take the same alphabet $X, S(\mathcal{A})=S\left(\mathcal{A}_{1}\right) \cup S\left(\mathcal{A}_{2}\right)$, the new start state to be $s_{0}\left(\mathcal{A}_{1}\right)$, the final states to be $F_{0}(\mathcal{A})=F_{0}\left(\mathcal{A}_{1}\right) \cup F_{0}\left(\mathcal{A}_{2}\right)$, the transition function to be $\delta_{1} \cup \delta_{2}$; and add additional arrows labelled $\varepsilon$ from all states of $F_{0}\left(\mathcal{A}_{1}\right)$ to $s_{0}\left(\mathcal{A}_{2}\right)$. Since $A_{1} A_{2}$ is $k$-reduced, the length of cancellations between the elements $a_{1} a_{2}$, where $a_{1} \in A_{1}, a_{2} \in A_{2}$ is bounded by $k$. Therefore, the set $U$ of words $u_{j} \in F(X)$ such that $a_{1}=b_{1} \circ u_{j}^{-1}$, $a_{2}=u_{j} \circ b_{2}$, and $a_{1} a_{2}=b_{1} \circ b_{2}$, where $a_{i} \in A_{i}, b_{i} \in F(X)$, is finite. Since $\mathcal{A}_{1}$ is a finite state automaton, for every $u_{j} \in U$ there is a finite set $P_{u_{j}}=\left\{p_{u_{j}} \in\right.$ $S\left(\mathcal{A}_{1}\right): u_{j}^{-1}$ is readable from $p_{u_{j}}$ to $f_{0}$ for some $\left.f_{0} \in F_{0}\left(\mathcal{A}_{1}\right)\right\}$; by the same argument the set $Q_{u_{j}}=\left\{q_{u_{j}} \in S\left(\mathcal{A}_{2}\right): u_{j}\right.$ is readable from $s_{0}\left(\mathcal{A}_{2}\right)$ to $\left.q_{u_{j}}\right\}$ is finite. Add $\varepsilon$-transitions from the states of $P_{u_{j}}$ to the states of $Q_{u_{j}}$ for all $u_{j}$. Clearly, $\overline{A_{1} A_{2}} \subseteq L(\mathcal{A})$ by construction, and moreover, $\overline{A_{1} A_{2}}=$ $L(\mathcal{A}) \cap F(X)$. Thus $\overline{A_{1} A_{2}}$ is an intersection of regular sets, so is itself regular. 
The double-based cone with bases $w_{1}, w_{2}$ is the set of all reduced words $w_{1} \circ f \circ w_{2}$ in $F(X)$, starting with $w_{1}$ and ending with $w_{2}$.

Corollary 3.15. Every double-based cone $C\left(w_{1}, w_{2}\right)$ in a finite rank free group is a regular set.

Proof. One can prove this statement using Benois' theorem, but we are interested in it's direct proof and corresponding automaton. Let $w_{1}=$ $x_{i_{1}} \cdots x_{i_{s}}, w_{2}=x_{j_{1}} \cdots x_{j_{t}}$. Then by definition an element $f \in C\left(w_{1}, w_{2}\right)$ is an $X$-reduced word of the form $f=x_{i_{1}} \cdots x_{i_{s}} \circ y \cdots z \circ x_{j_{1}} \cdots x_{j_{t}}$, with $y \neq x_{i_{s}}^{-1}$, and $z \neq x_{j_{1}}^{-1}$. Thus, it is sufficient to prove the regularity of a cone with bases $x_{i_{s}}$ and $x_{j_{1}}$. Indeed, if $C\left(x_{i_{s}}, x_{j_{1}}\right)$ is regular, then so is $C\left(w_{1}, w_{2}\right)=x_{i_{1}} \cdots x_{i_{s-1}} \circ C\left(x_{i_{s}}, x_{j_{1}}\right) \circ x_{j_{2}} \cdots x_{j_{t}}$ as it is a concatenation of regular sets in $F(X)$. For notational simplicity we construct a deterministic automaton $\mathcal{A}$ recognizing the cone $C\left(x_{i_{s}}, x_{j_{1}}\right)$ in $F(X)=F(a, b)$, for bases equal to $a$ and $b$ respectively. An automaton $\mathcal{A}$ recognizing $C(a, b)$ is shown in Figure 3 (the tailed arrow corresponds to the initial state plus $a$-transition, and the final state is labelled by double circle).

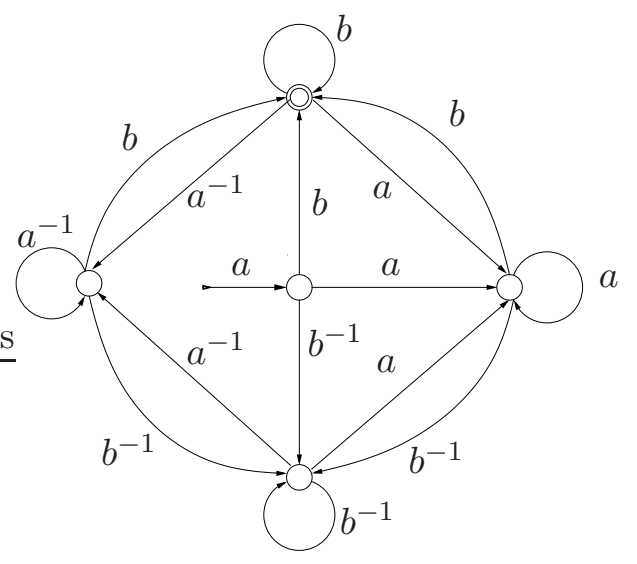

Figure 3: The automaton $\mathcal{A}$ recognizing the cone $C(a, b)$.

If now the rank of the free group $F$ is greater than $2, \mathcal{A}$ can be easily modified to recognize $C(a, b)$ in $F=F(X)$. Namely, it is necessary to add a new state $q_{x}$ corresponding to each element of $X \cup X^{-1}$ (except $a^{ \pm 1}, b^{ \pm 1}$ ). From every new state $q_{x}$ we add a $y$-transition to every other state $q_{y}$, including $q_{x}$, but excluding $q_{x^{-1}}$. The arrows from basis $a$ and to basis $b$ can be added in similar way. 
Theorem 3.16. Let $C$ be a finite rank subgroup of a free group $F(X)$. Then the set of all $X$-reduced words representing elements of $C f C$ is regular in $F(X)$. Moreover, an automaton which accepts this set can be constructed effectively by $C$ and $f$ if $C$ is $f$-malnormal, or by $C, f$ and a relative Schreier transversal $T$ of $C$ with respect to $C_{f}$, otherwise.

Proof. If $C_{f}=1$, then every $X$-reduced word in $C f C$ has a unique representation in terms of this complex, by Lemma 3.4. If $C_{f} \neq 1$, then every word in $C f C$ can be represented by an element of the complex $C f T$, for a relative Schreier transversal $T$ of $C$ with respect to $C_{f}$, by Lemma 3.10, In both cases the sets $C f C$ and $C f T$ are regular: it follows either from Benois' theorem, or from the following direct construction. The sets $C f C$ and $C f T$ are $k$-reduced in $F(X)$, by Theorem 3.12, Further, since $C, f C$ and $f T$ are regular in $F(X)$ and corresponding automata can be constructed effectively (see, for example, [8]), the result follows from Remark 3.14 and it's proof.

\section{Acknowledgments}

The first author is grateful to A. Duncan, A. A. Klyachko and V. A. Romankov for useful remarks. The second author was partially supported by RFFI grant 11-01-00081.

\section{References}

[1] G. Baumslag, A. G. Miasnikov, V. N. Remeslennikov, Malnormality is decidable in free groups, Intern. J. Algebra Comput. 9(6) (1999) 687-692.

[2] M. Benois, Parties rationnelles du groupe libre, C.R. Acad. Sci. Paris, Ser. A, 269 (1969)

[3] M. Bestvina, M. Feighn, A combination theorem for negatively curved groups, Intern. J. Diff. Geom. 35(1) (1992) 85-101.

[4] A. V. Borovik, A. G. Myasnikov and V. N. Remeslennikov, The Conjugacy Problem in Amalgamated Products I: Regular Elements and Black Holes, Intern. J. Algebra Comput. 17(7) (2007) 1301 - 1335.

[5] M. Bridson and D. Wise, Malnormality is undecidable in hyperbolic groups, Israel J. Math. 124 (2001) 313-316. 
[6] I. Chiswell, Abstract length functions in groups, Math. Proc. Cambridge Phil. Soc. 80 (1976) 451-463.

[7] D. Epstein, J. Cannon, D. Holt, S. Levy, M. Paterson and W. Thurston, Word Processing in Groups (Jones and Bartlett, Boston, 1992).

[8] E. Frenkel, A. G. Myasnikov and V. N. Remeslennikov, Regular sets and counting in free groups, in Combinatorial and Geometric Group Theory, Series "Trends in Mathematics", (Birkhauser Verlag Basel/Switzerland, 2010), pp. 93-118.

[9] E. Frenkel, A. G. Myasnikov and V. N. Remeslennikov, Amalgamated products of groups: measures of random normal forms, Fund. Appl. Math. 16(8) (2010) 189-221.

[10] M. Gromov, Hyperbolic groups, in Essays in Group Theory, Mathematical Sciences Research Institute Publications, Vol. 8 (Springer-Verlag, Berlin, 1987), pp. 75-263.

[11] T. Jitsukawa, Malnormal subgroups of free groups, in Computational and statistical group theory, Series on Contemporary Mathematics, Vol. 298, Amer. Math. Soc., Providence, RI, (2002) pp. 83-95.

[12] I. Kapovich and A. G. Myasnikov, Stallings foldings and subgroups of free groups, J. Algebra 248 (2002) 608-668.

[13] O. Kharlampovich and A. Myasnikov, Hyperbolic groups and free constructions, Trans. Amer. Math. Soc. 350(2) (1998) 571-613.

[14] R. Lyndon, Length functions in groups, Math. Scand., 12 (1963) 209 234.

[15] R. C. Lyndon and P. Schupp, Combinatorial group theory (Ergebnisse der Mathematik und ihrer Grenzgebiete Vol. 89, Springer-Verlag, Berlin, Heidelberg, New York, 1977).

[16] K. V. Mikhajlovskii and A. Yu. Ol'shanskii, Some constructions relating to hyperbolic groups, in Geometry and Cohomology in Group Theory, London Math. Soc. Lecture Notes Series, Vol. 252 (Cambridge University Press, Cambridge, 1998), pp. 263-290.

[17] N. W. M. Touikan, A Fast Algorithm for Stallings' Folding Process, Intern. J. Algebra Comput. 16(6) (2006) 1031-1046. 